\title{
Hawking Radiation of Black Holes in Infrared Modified Hořava-Lifshitz Gravity
}

\author{
Jun-Jin Peng ${ }^{\dagger}$, Shuang-Qing Wu \\ College of Physical Science and Technology, Central China Normal University, \\ Wuhan, Hubei 430079, People's Republic of China \\ E-Mail: pengjjph@163.com ${ }^{\dagger}$
}

\begin{abstract}
We study the Hawking radiation of the spherically symmetric, asymptotically flat black holes in the infrared modified Hořava-Lifshitz gravity by applying the methods of covariant anomaly cancellation and effective action, as well as the approach of Damour-RuffiniSannan's. These black holes behave as the usual Schwarzschild ones of the general relativity when the radial distance is very large. We also extend the method of covariant anomaly cancellation to derive the Hawking temperature of the spherically symmetric, asymptotically AdS black holes that represent the analogues of the Schwarzschild AdS ones.
\end{abstract}

\section{Introduction}

Recently, Hořava proposed a power counting renormalizable theory for the (3+1)-dimensional quantum gravity [1], motivated by the Lifshitz model in condensed matter physics. This theory, called as Hořava-Lifshitz (HL) gravity, is believed to be the potential ultraviolet (UV) completion of general relativity. In the infrared (IR) limit (setting the dynamical coupling constant $\lambda=1$ in the action), it recovers general relativity. Some important characters of the HL gravity are that the Lorentz invariance is violated at the UV level and the scaling between time and space is also anisotropic. The latter indicates that this model is not invariant under the full diffeomorphism group of general relativity but has the foliation preserving diffeomorphism invariance for generic values of $\lambda$. Inspired by the HL gravity, a 
great number of works have appeared to investigate the classical solutions [2, 3, 4, 5], the black hole physics [6, 7, 8] and other aspects [9, 10, 11].

In order to reduce the number of the independent coupling constants, in [1], the detailed balance condition was imposed. One result from the imposition is that the generic IR vacuum of the HL gravity is not a Minkowski vacuum but the one in anti-de Sitter background. Besides, in the IR limit, both the Newton constant and the speed of light are dependent on the cosmological constant. For the aim to make the HL gravity recover the Minkowski vacuum in the IR limit, the theory was modified by adding a term " $\mu^{4} R$ " [2] to the original action in [1]. Such a modification softly breaks the detailed balance condition and changes the IR properties. But the ones at the UV level are not affected. In terms of the IR modified HL gravity, a static, spherically symmetric black hole solution was presented in [2]. This black hole behaves like the standard Schwarzschild one when the radial distance is sufficiently large. In [4], the author obtained the black hole and cosmological solution that is very similar with the usual Schwarzschild-AdS black hole at long distances.

On the other hand, Hawking radiation [12] has been regarded as a very attractive quantum phenomenon of black holes or other geometric backgrounds with an event horizon. It may open way for understanding the quantum gravity. Since this quantum effect was discovered more than thirty years ago, several different approaches have been developed to derive it. One of them is the gravitational and gauge anomaly cancellation method recently proposed by Robinson and Wilczek (RW) [13]. They first obtained the Hawking temperature of the Schwarzschild black hole by considering the gravitational anomaly. Subsequently, taking into account both gravitational anomaly and gauge anomaly, Iso, Umetsu and Wilczek extended RW's method to the charged black hole [14] and the rotating black hole [15]. This anomaly cancellation method treats the Hawking radiation as a compensation of the quantum anomaly that breaks the classical symmetry near the horizon. In terms of the two dimensional effective metric, which is obtained due to the fact that an infinite collection of (1+1)-dimensional fields can effectively describe the quantum field near the horizon of the original higher dimensional space-time, one can derive the fluxes of charges (if there exists gauge field) and energy momentum tensor by solving the Ward identities under proper boundary conditions associated with the Unruh vacuum. These fluxes are identified with the Hawking ones. Following RW's method, a lot of works have appeared to investigate the Hawking radiation of black objects in various dimensions [16, 17, 18, 19, 20, 21, 22, 23, 24, 25, 26]. 
There are two forms of anomalies. One is consistent and the other is covariant. For the original method proposed by RW in [13, 14], the expressions of chiral anomalies take the consistent forms, but the boundary condition to fix the arbitrary parameters is covariant. In [23], it was argued that the anomaly cancellation method can be unified in terms of the single covariant expressions, namely, the fluxes of charges and energy momentum tensor can be obtained via covariant gauge anomaly and covariant gravitational anomaly, together with the vanishing of the covariant current at the event horizon. Such an argument makes the anomaly cancellation method more economical and conceptually cleaner. Since then, on basis of the development in [23, Hawking radiation of black strings in various dimensions was discussed [24]. Other extensions can be found in [25, 26].

It is worth noting that the boundary condition plays a crucial role in fixing the Hawking fluxes for both the RW's original method and the one of the covariant anomaly cancellation developed later. In fact, by imposing proper boundary condition at the event horizon only, Banerjee and Kulkarni [27] made use of the covariant current and energy momentum tensor, generated from the two dimensional effective action [28], to derive the Hawking fluxes of the spherically symmetric charged black holes. Their results are in agreement with those calculated via the approaches of Unruh vacuum [15] and the trace anomaly [29]. Some applications of the covariant effective action method generalized by Banerjee and Kulkarni appear in [30]. In our work [21], this method has been extended to reproduce the Hawking fluxes of the Schwarzschild black holes in the isotropic coordinates where the determinant of the metric vanishes at the horizon.

So far although some properties of the black holes in HL gravity have been investigated, the study on their Hawking radiation is still vacant. In this paper, we shall fill this gap by applying the methods of covariant anomaly cancellation and the covariant effective action to exploit the Hawking radiation of the spherically symmetric, asymptotically flat black holes [2] in the IR modified HL gravity. By comparison, we will also calculate their Hawking temperature via the method of Damour-Ruffini-Sannan (DRS) [31]. Besides, we shall study the Hawking radiation of the spherically symmetric, asymptotically AdS black holes [4] through the covariant anomaly cancellation method. The remainder of the present paper is organized as follows. In section 2, we shall briefly review the IR modified HL gravity and the spherically symmetric, asymptotically flat black hole solutions in [2]. In section [3, we will use the covariant anomaly cancellation method to derive the Hawking temperature of these black holes. By applying the covariant effective action approach, we reproduce the 
same Hawking fluxes as those obtained via the method of covariant anomaly cancellation in section 4. In section 5, we compute the Hawking temperature of the asymptotically flat black holes via the DRS approach. In section 6, the analysis in section 3 is extended to study the Hawking radiation of the spherically symmetric black holes with cosmological constant [4]. We present our conclusions in the last section.

\section{Black Holes in Infrared Modified Hořava-Lifshitz Gravity}

Let us start with the metric ansatz in the four dimensional HL gravitational theory. Using the ADM-like decomposition and introducing the lapse function $N$, the shift vector $N_{i}$ and the spatial metric $g_{i j}$, we have

$$
d s_{4}^{2}=-\left(N^{2}-N^{k} N_{k}\right) d t^{2}-2 g_{i j} N^{i} d t d x^{j}+g_{i j} d x^{i} d x^{j} .
$$

In what follows, the action with IR modification takes the form [2]

$$
\begin{aligned}
I= & \int d t d^{3} x \sqrt{g} N\left[\frac{2}{\kappa^{2}}\left(K_{i j} K^{i j}-\lambda K^{2}\right)+\frac{\kappa^{2} \mu^{2} \Lambda_{W}}{8(1-3 \lambda)}\left(R-3 \Lambda_{W}+\frac{1-4 \lambda}{4 \Lambda_{W}} R^{2}\right)+\mu^{4} R\right. \\
& \left.-\frac{\kappa^{2}}{2 w^{4}}\left(C_{i j}-\frac{\mu w^{2}}{2} R_{i j}\right)\left(C^{i j}-\frac{\mu w^{2}}{2} R^{i j}\right)\right],
\end{aligned}
$$

where $\lambda, \mu, w, \kappa$ are constant parameters, the extrinsic curvature $K_{i j}$ is defined by

$$
K_{i j}=\frac{1}{2 N}\left[\partial_{t} g_{i j}-2 \nabla_{(i} N_{j)}\right],
$$

and the Cotton tensor $C^{i j}$ is read off as

$$
C^{i j}=\frac{\epsilon^{i k l}}{\sqrt{g}} \nabla_{k}\left(R^{j}{ }_{l}-\frac{1}{4} R \delta^{j}{ }_{l}\right) .
$$

Compared with the original action in [1], the added term " $\mu^{4} R$ " in the action (2.2) softly violates the detailed balance condition and modifies the IR properties of the HL gravity [2], however, it does not amend the UV ones. Particularly, in the IR limit by setting

$$
\begin{aligned}
\lambda & =1, \quad \Lambda_{W}=0, \\
c^{2} & =\frac{\kappa^{2} \mu^{4}}{2}, \quad G_{N}=\frac{\kappa^{2}}{32 \pi c},
\end{aligned}
$$

the action (2.2) reduces to the standard Einstein-Hilbert one in the ADM formalism

$$
I_{E H}=\frac{1}{16 \pi G_{N}} \int d^{4} x \sqrt{g} N\left(K_{i j} K^{i j}-K^{2}+R\right),
$$


where we have introduced the coordinate $x^{0}=c t$. Due to the variations to $N, N_{i}$ and $g_{i j}$, one can get the equations of motion. The similar motion equations can be found in 3 . When $\lambda=1$ and $\Lambda_{W}=0$, a static, spherically symmetric black hole solution that satisfies the full set of motion equations is presented as [2]

$$
\begin{aligned}
& d s^{2}=-f(r) d t^{2}+\frac{d r^{2}}{f(r)}+r^{2}\left(d \theta^{2}+\sin ^{2} \theta d \phi^{2}\right), \\
& f(r)=\frac{2 r^{2}+2 P^{2}-4 r M}{r^{2}+2 P^{2}+\sqrt{r^{4}+8 P^{2} M r}},
\end{aligned}
$$

where $P^{2}=\kappa^{2} /\left(32 \mu^{2}\right)$, and $M$ is an integration constant. This black hole is formally different from the usual Schwarzschild black hole. However, for $r \gg 2\left(M P^{2}\right)^{(1 / 3)}$, we find that $f(r)$ takes the form

$$
f(r)=1-\frac{2 M}{r}+\mathcal{O}\left(\frac{1}{r^{4}}\right)
$$

which implies that the solution (2.7) behaves like the usual Schwarzschild black hole in general relativity when $r$ is sufficiently large. If $P \rightarrow 0$ and $r$ is finite, we have $f(r)=$ $1-2 M / r$, meaning that Eq. (2.7) exactly coincides with the Schwarzschild black hole. Its two event horizons that are very similar with those of the Reissner-Nordström black hole are

$$
r_{ \pm}=M \pm \sqrt{M^{2}-P^{2}}
$$

where $M \geq|P|$ to avoid the naked singularity. $M=|P|$ is the extremity condition. Some properties of the black hole (2.7) have been investigated in [6].

By virtue of the surface gravity formula, the Hawking temperature of the black hole (2.7) is given by

$$
T_{H}=\left.\frac{1}{4 \pi} \partial_{r} f(r)\right|_{r_{+}}=\frac{r_{+}^{2}-P^{2}}{4 \pi\left(r_{+}^{3}+2 P^{2} r_{+}\right)} .
$$

Obviously, when $M=|P|$, the temperature for the extremal black hole vanishes. The ADM mass is $M$. If we naively use the area law to calculate the entropy, we obtain the evaluation that does not fulfill the first law of black hole thermodynamics. To keep the differential form of the first law hold, i.e. $d M=T_{H} d S$, we have to set the entropy $S=\pi r_{+}^{2}+4 \pi P^{2} \ln \left(r_{+}\right)+S_{0}$ [7], where $S_{0}$ is an arbitrary constant irrelevant with $r_{+}$. However, in this case, the integral form of the first law is not satisfied. Maybe we can modify both the mass and entropy to guarantee that the differential and integral forms of the fist law hold at the same time. We do not discuss this point in the present paper. In what follows, we shall focus on 
reproducing the temperature (2.10) via the methods of gravitational anomaly cancellation and the effective action, as well as the DRS approach.

\section{Hawking radiation via covariant gravitational anomaly}

In this section, we will exploit the Hawking radiation of the black hole (2.7) via the covariant gravitational anomaly cancellation method [23] developed due to [13, 14]. Before doing this, we first give a brief review of this method. Considering a complex scalar field in the background of the black hole, since the field near the horizon can be effectively described by an infinite collection of (1+1)-dimensional fields, it is feasible for us to treat the original higher dimensional theories as a set of scalar fields in the background of $(1+1)$-dimensional spacetime. Near the horizon of this two dimensional reduced space-time, there exist outgoing modes and ingoing modes. If we omit the classically irrelevant ingoing modes, the $(1+1)$ dimensional effective field theory becomes chiral, which leads to gravitational anomaly. In order to cancel this anomaly, a compensating current that is identified to the Hawking flux of energy-momentum arises. It is worth noting that one has to impose proper boundary conditions that compatible with the Unruh vacuum to fix the Hawking flux completely.

Now we discuss the dimension reduction by considering the action of a massless scalar fields in background of the black hole (2.7). Performing the partial wave decomposition $\varphi=\sum_{l m} \varphi_{l m}(t, r) Y_{l m}(\theta, \phi)$, where $Y_{l m}$ are the spherical harmonics, and only keeping the dominant terms near the horizon,

$$
\begin{aligned}
S[\varphi] & =-\frac{1}{2} \int d^{4} x \sqrt{-g} g^{\mu \nu} \partial_{\mu} \varphi \partial_{\nu} \varphi \\
& =\frac{1}{2} \int d t d r d \theta d \phi \sin \theta \varphi\left\{-\frac{r^{2}}{f} \partial_{t}^{2}+\partial_{r}\left(r^{2} f \partial_{r}\right)+\Delta_{\Omega}\right\} \varphi \\
& =\frac{1}{2} \sum_{l m} \int d t d r\left(r^{2}\right) \varphi_{l m}\left\{-\frac{1}{f} \partial_{t}^{2}+\partial_{r}\left(f \partial_{r}\right)\right\} \varphi_{l m},
\end{aligned}
$$

where $\Delta_{\Omega}$ is the angular Laplace operator, and the last equation is obtained by taking the near horizon limit. The above equation implies that the scalar field in the original $(3+1)-$ dimensional space-time can be effectively described by an infinite set of two dimensional fields in the background of the metric

$$
d s^{2}=-f(r) d t^{2}+\frac{d r^{2}}{f(r)}
$$


together with the dilaton field $\Psi=r^{2}$, whose contribution can be neglected, since the reference space-time is static. Obviously, the effective metric (3.2) is just the $(t, r)$ part of the original metric (2.7).

Near the horizon, if we omit the quantum effect of the ingoing modes, the effective field theory will exhibit a gravitational anomaly. For the right-handed fields, the $(1+1)$ dimensional covariant gravitational anomaly has the form

$$
\nabla_{\mu} T_{\nu}^{\mu}=\frac{-1}{96 \pi} \sqrt{-g_{2}} \epsilon_{\mu \nu} \partial^{\mu} R=\frac{1}{\sqrt{-g_{2}}} \partial_{\mu} N_{\nu}^{\mu}
$$

where $\epsilon_{\mu \nu}$ is the two dimensional tensor density with $\epsilon^{r t}=\epsilon_{t r}=-1, R$ is the scalar curvature of the two dimensional space-time, and $g_{2}$ is the determinant of the two dimensional metric. In terms of the effective metric (3.2), the anomaly is time-like, i.e. $\nabla_{\mu} T_{r}^{\mu}=0$, and

$$
N_{t}^{r}=\frac{1}{192 \pi}\left(2 f f^{\prime \prime}-f^{\prime 2}\right)
$$

where and in what follows, the prime denotes the derivative to the radial coordinate. Introducing two scalar functions $\Theta(r)=\Theta\left(r-r_{+}-\varepsilon\right)$ and $H(r)=1-\Theta(r)$, where the limit $\varepsilon \rightarrow 0$ will be taken ultimately, the total energy-momentum tensor can be written as

$$
T_{\nu}^{\mu}=T_{(O) \nu}^{\mu} \Theta(r)+T_{(H) \nu}^{\mu} H(r),
$$

in which $T_{(O) \nu}^{\mu}$, localized in the region outside the horizon $\left(r>r_{+}+\varepsilon\right)$, is covariantly conserved, while $T_{(H) \nu}^{\mu}$ that is defined in the near-horizon region $\left(r_{+}<r<r_{+}+\varepsilon\right)$ satisfies the anomalous Eq. (3.3). Solving both the equations, we have

$$
\begin{aligned}
& T_{(O) t}^{r}=a_{O}, \\
& T_{(H) t}^{r}=a_{H}+N_{t}^{r}(r)-N_{t}^{r}\left(r_{+}\right),
\end{aligned}
$$

where $a_{O}$ and $a_{H}$ are two integration constants, and $a_{O}$ is the value of the energy flow at infinity. In order to fix it completely, firstly we take into account the $\nu=t$ component of Eq. (3.3), which reads

$$
\nabla_{\mu} T_{t}^{\mu}=\partial_{r} T_{t}^{r}=\partial_{r}\left[N_{t}^{r} H(r)\right]+\left[T_{(O) t}^{r}-T_{(H) t}^{r}+N_{t}^{r}\right] \delta\left(r-r_{+}\right) .
$$

The first term in the above equation should be cancelled by the ingoing modes . For the sake of keeping the energy-momentum tensor anomaly free, the second term must vanish at the horizon. Therefore, we obtain

$$
a_{O}=a_{H}-N_{t}^{r}\left(r_{+}\right)
$$


However, this equation is not sufficient to fix energy flow $a_{O}$ completely. One has to impose a regular boundary condition corresponding to the Unruh vacuum. Such a constraint requires the covariant energy-momentum tensor $T_{\nu}^{\mu}$ to vanish at the horizon, which yields $a_{H}=0$. Thus the total energy flux at infinity is read off as

$$
a_{O}=-N_{t}^{r}\left(r_{+}\right)=\frac{f^{\prime 2}\left(r_{+}\right)}{192 \pi}=\frac{1}{192 \pi}\left(\frac{r_{+}^{2}-P^{2}}{r_{+}^{3}+2 P^{2} r_{+}}\right)^{2} .
$$

The energy fluxes (3.9), obtained via the covariant gravitational anomaly, is compatible with the Hawking ones. To see this, we calculate the Hawking fluxes by considering the fermionic Plack distribution $N(\omega)=1 /\left(e^{\omega / T_{H}}+1\right)$ for blackbody radiation in the general spherically symmetric black hole background. With such a distribution, the Hawking fluxes of the energy-momentum tensor is computed as

$$
F_{M}=\int_{0}^{\infty} \frac{\omega}{\pi\left(e^{\omega / T_{H}}+1\right)} d \omega=\frac{\pi}{12} T_{H}^{2}
$$

Substituting the Hawking temperature (2.10) into Eq. (3.10), we have $a_{O}=F_{M}$. This implies that we can also derive the same temperature as Eq. (2.10) via the method of the covariant gravitational anomaly cancellation.

\section{Hawking radiation through the method of covariant effec- tive action}

It has been shown that the quantum field can be effectively described by an infinite collection of $(1+1)$-dimensional fields with the metric (3.2) in the near-horizon region of the black hole (2.7). Thus we shall employ the covariant effective action method [27] to calculate the Hawking fluxes in terms of the background of the effective metric (3.2) in this section. Our calculations only involve the gravitational part of the effective action since there only exists gravitational anomaly near the horizon. Some results can be found in our work [21]. Due to

the effective action method, the Hawking fluxes are associated with the $(r, t)$-component of the covariant anomalous energy-momentum tensor near the horizon, which can be derived by varying the effective action. With help of the boundary condition that the covariant energy-momentum tensor vanishes at the horizon, one obtains the Hawking flux that is equal to the $(r, t)$-component of the energy-momentum tensor at infinity $(r \rightarrow \infty)$. 
In the (1+1)-dimensional black hole background, the covariant energy momentum tensor is given by 28 ]

$$
T_{\nu}^{\mu}=-\frac{1}{192 \pi}\left(D^{\mu} \psi D_{\nu} \psi-2 D^{\mu} D_{\nu} \psi+2 \delta^{\mu}{ }_{\nu} R\right) .
$$

For Eq. (4.1), the auxiliary field $\psi$ obeys the equation

$$
\nabla_{\mu} \nabla^{\mu} \psi=R=-f^{\prime \prime}
$$

which vanishes at infinity, while the chiral covariant differential operator $D_{\mu}$ is defined by

$$
D_{\mu}=\nabla_{\mu}+\sqrt{-g} \epsilon_{\mu \nu} \nabla^{\nu}=\sqrt{-g} \epsilon_{\mu \nu} D^{\nu}
$$

One can verify that the covariant energy-momentum tensor (4.1) satisfies both the covariant anomaly equation (3.3) and the conformal trace anomaly equation

$$
T_{\mu}^{\mu}=-\frac{R}{48 \pi}
$$

For the two-dimensional effective metric (3.2), the equation of motion (4.2) for the auxiliary field $\psi$ becomes

$$
-\frac{1}{f} \partial_{t}^{2} \psi+\partial_{r}\left(f \partial_{r} \psi\right)=-\partial_{r}^{2} f
$$

The general solution of this equation is

$$
\begin{aligned}
\psi & =a t+\int \frac{b-f^{\prime}}{f} d r=a t-\ln (f)+b \int \frac{d r}{f}, \\
D_{t} \psi & =-D^{r} \psi=a-b+f^{\prime},
\end{aligned}
$$

where $a$ and $b$ are constants to be specified by imposing appropriate boundary condition. Therefore, the $(r, t)$-component of the covariant energy momentum tensor (4.1) can be computed as

$$
T_{t}^{r}=\frac{1}{192 \pi}\left[(a-b)^{2}-f^{\prime 2}+2 f f^{\prime \prime}\right] .
$$

As before, we still choose the boundary condition that the covariant energy momentum tensor vanishes at event horizon. Thus we have

$$
(a-b)^{2}=f^{\prime 2}\left(r_{+}\right)
$$

In [27], it has been shown that the energy flux is given by the asymptotical limit of the anomaly free energy momentum tensor. For the effective metric (3.2), since $f^{\prime}(\infty)=0$ and 
$f^{\prime \prime}(\infty)=0$, the Eq. (3.3) becomes $\nabla_{\mu} T_{\nu}^{\mu} \rightarrow 0$ when $r$ goes to infinity, which implies that the energy momentum tensor is anomaly free at infinity. Therefore, the energy flux reads

$$
T_{t}^{r}(r \rightarrow \infty)=\frac{f^{\prime 2}\left(r_{+}\right)}{192 \pi}=\frac{1}{192 \pi}\left(\frac{r_{+}^{2}-P^{2}}{r_{+}^{3}+2 P^{2} r_{+}}\right)^{2}
$$

This energy flux agrees with Eq. (3.9) got via the method of covariant anomaly cancellation.

Besides, we can also evaluate the energy flux by calculating the anomaly free energy momentum tensor outside the horizon, which reads [28]

$$
T_{(O) \nu}^{\mu}=-\frac{1}{96 \pi}\left(4 \delta^{\mu}{ }_{\nu} R-4 \nabla^{\mu} \nabla_{\nu} \psi+2 \nabla^{\mu} \psi \nabla_{\nu} \psi-\delta^{\mu}{ }_{\nu} \nabla^{\sigma} \psi \nabla_{\sigma} \psi\right)
$$

This anomaly-free energy momentum satisfies $\nabla_{\mu} T_{(O) \nu}^{\mu}=0$. Its $(r, t)$ component is

$$
T_{(O) t}^{r}=-\frac{a b}{48 \pi} .
$$

Here we do not give the other components of the energy momentum tensor $T_{(O) \nu}^{\mu}$. All the components coincide with their corresponding ones obtained via the Unruh vacuum approach [15] if we still choose the boundary condition that the covariant energy momentum disappears at the horizon. Since Eq. (3.3) vanishes at infinity, Eq. (4.11) must be in agreement with Eq. (4.7) when we take the asymptotical limit. Thus we have $a=-b$. With the help of Eq. (4.8), we obtain

$$
\begin{aligned}
& a=f^{\prime}\left(r_{+}\right) / 2, \quad b=-f^{\prime}\left(r_{+}\right) / 2, \\
& a=-f^{\prime}\left(r_{+}\right) / 2, \quad b=f^{\prime}\left(r_{+}\right) / 2 .
\end{aligned}
$$

Substituting each of these equations into Eq. (4.11), we get the energy flux that takes the same form as Eq. (4.9). Looking through the whole procedure, we find that the asymptotical behaviour of $f(r)$ plays a key role in our derivation. If $f^{\prime 2}-2 f f^{\prime \prime}$ is divergent when $r \rightarrow \infty$, all of the derivation is invalid. For example, our calculation in this section fails to give the energy flux of the Schwarzschild AdS black hole. Finally, from the energy flux, we can obtain the Hawking temperature in agreement with Eq. (2.10).

\section{Hawking radiation via the DRS approach}

In this section, we will make use of the DRS approach [31] to investigate Hawking radiation of the black hole (2.7) in terms of the $(1+1)$-dimensional effective metric (3.2). This 
approach has also been employed to study Hawking radiation from the Reissner-Nordström black hole with a global monopole in [19]. We now take into account the Klein Gordon equation of a complex scalar field $\Phi$ with mass $\mu$, which reads

$$
-\frac{1}{f} \partial_{t}^{2} \Phi+\partial_{r}\left(f \partial_{r} \Phi\right)-\mu^{2} \Phi=0
$$

Separating the scalar field $\Phi$ as $\Phi(t, r)=R(r) e^{-i \omega t}$ and performing the coordinate transformation $r_{*}=\int d r / f$, the radial equation is given by

$$
\frac{d^{2} R(r)}{d r_{*}^{2}}+\left(\omega^{2}-\mu^{2} f\right) R(r)=0
$$

Near the horizon, $f(r) \rightarrow 0$. Thus the scalar field $\Phi$ can be solved as

$$
\begin{aligned}
\Phi_{\text {in }} & =e^{-\mathrm{i} \omega\left(t+r_{*}\right)}, \\
\Phi_{\text {out }} & =e^{-\mathrm{i} \omega\left(t-r_{*}\right)}=\Phi_{i n} e^{2 \mathrm{i} \omega r_{*}},
\end{aligned}
$$

where $\Phi_{i n}$ is the ingoing wave solution, which is analytic at the horizon, while $\Phi_{\text {out }}$, the outgoing wave solution, is logarithmically singular at the horizon. To see this, by virtue of the relation $r_{*} \approx \ln \left(r-r_{+}\right) /(2 \kappa)$ near the horizon, where $\kappa=f^{\prime}\left(r_{+}\right) / 2$ is the surface gravity of the black hole (2.7), we can reexpress the outgoing wave solution as

$$
\Phi_{\text {out }} \approx \Phi_{\text {in }}\left(r-r_{+}\right)^{\mathrm{i} \omega / \kappa} \quad\left(r>r_{+}\right) .
$$

However, it can be analytically continued to

$$
\widetilde{\Phi}_{\text {out }}=\Phi_{\text {in }}\left(r_{+}-r\right)^{\mathrm{i} \omega / \kappa} e^{\pi \omega / \kappa} \quad\left(r<r_{+}\right)
$$

from the outside of the hole into the inside hole along the lower $r$-plane. Therefore, we obtain the relative scattering probability at the horizon

$$
\left|\frac{\Phi_{\text {out }}}{\widetilde{\Phi}_{\text {out }}}\right|^{2}=e^{-2 \pi \omega / \kappa} .
$$

Following the DRS approach proposed in [31, the thermal spectrum of the particles radiating from the black hole can be given by $N(\omega)=1 /\left(e^{2 \pi \omega / \kappa}-1\right)$ for scalar particles. From the thermal spectrum, we can obtain the Hawking temperature $T_{H}=f^{\prime}\left(r_{+}\right) /(4 \pi)$, which takes the same form as Eq. (2.10). 


\section{Hawking radiation of black holes with cosmological con- stant}

In this section, we shall extend the gravitational anomaly cancellation method to exploit the Hawking radiation of the static, spherically symmetric black hole with cosmological constant in the IR Modified HL Gravity. This black hole solution, which fulfills the motion equations generated from the action (2.2) with the coupling constant $\lambda=1$, is given by [4]

$$
\begin{aligned}
d s^{2} & =-h(r) d t^{2}+\frac{d r^{2}}{h(r)}+r^{2}\left(d \theta^{2}+\sin ^{2} \theta d \phi^{2}\right), \\
h(r) & =\frac{1}{2 P^{2}}\left[2 P^{2}+r^{2}\left(1-2 \Lambda_{W} P^{2}\right)-\sqrt{r^{4}\left(1-4 \Lambda_{W} P^{2}\right)+4 r m P^{4}}\right],
\end{aligned}
$$

where $m$ is an integration constant. Choosing proper values of $\Lambda_{W}, P$ and $m$, the solution (6.1) can recover some known solutions. We first set $\Lambda_{W}=0$ and $m=2 M / P^{2}$. In such a case, the solution (6.1) becomes the one (2.7), which agrees with the usual Schwarzschild black hole. When $\Lambda_{W}<0$, for $r \gg\left[m P^{4} /\left(1-4 P^{2} \Lambda_{W}\right)\right]^{1 / 3}$ and $m=2 M / P^{2}$, Eq. (6.1) reduces to the standard Schwarzschild-AdS black hole except for a factor " $\left|2 \Lambda_{W} P^{2}\right|$ " [4]. Besides, in this case, if $P \rightarrow \infty$ and $m=\alpha^{2} \sqrt{-\Lambda_{W}}$, Eq. (6.1) takes the same form as that in [3]. Finally, by setting $\Lambda_{W}>0$ and performing the transformation $\mu \rightarrow i \mu, w^{2} \rightarrow-i w^{2}$, one can obtain the usual Schwarzschild-dS-like solution.

Since the analysis via the anomaly cancellation approach here is parallel with what we have performed in the section (3), we only present some main results. By the dimension reduction technique, we have the two dimensional effective metric

$$
d s^{2}=-h(r) d t^{2}+\frac{d r^{2}}{h(r)} .
$$

In terms of this effective metric, the Hawking flux can be computed as

$$
a_{O}=\frac{h^{\prime 2}\left(r_{+}\right)}{192 \pi}=\frac{1}{192 \pi}\left\{\frac{3 \Lambda_{W}^{2} P^{2} r_{+}^{4}+\left(1-2 P^{2} \Lambda_{W}\right) r_{+}^{2}-P^{2}}{r_{+}\left[2 P^{2}+\left(1-2 P^{2} \Lambda_{W}\right) r_{+}^{2}\right]}\right\}^{2},
$$

where $r_{+}$, the largest root of the equation $h(r)=0$, is the outside horizon of the black hole (6.1). From the Hawking flux, we obtain the Hawking temperature, which reads

$$
T_{H}=\frac{3 \Lambda_{W}^{2} P^{2} r_{+}^{4}+\left(1-2 P^{2} \Lambda_{W}\right) r_{+}^{2}-P^{2}}{4 \pi r_{+}\left[2 P^{2}+\left(1-2 P^{2} \Lambda_{W}\right) r_{+}^{2}\right]} .
$$

This temperature coincides with the one derived by virtue of the surface gravity formula. In particular, when $\Lambda_{W}=0$, it becomes that of the asymptotically flat black hole (2.7). 
Besides, we can also derive the same temperature via the DRS method following the above section.

\section{Summary}

In this paper, we have extended the methods of covariant anomaly cancellation and the effective action to derive the Hawking temperature of the black holes [2] in the IR modified Hořava-Lifshitz gravity. These black holes are the spherically symmetric, asymptotically flat solutions when the coupling constant $\lambda=1$, namely, the HL gravity returns to general relativity. They are formally different from the usual Schwarzschild black holes but agree with the Schwarzschild ones when the radial distance is sufficiently large. The crucial points of both the two methods are the dimension reduction and the appropriate boundary condition. Using the dimension reduction technique that the complex scalar field in the background of the higher dimensional spacetime can be effectively described by an infinite collection of two dimensional scalar fields, we obtained the two dimensional effective metric. On basis of the effective metric, imposing the boundary condition that the fluxes of the energy momentum tensor vanishes at the event horizon, we applied both the methods to calculate the Hawking fluxes. The values obtained via these two methods are equal. Our results further support Hawking radiation is a universal quantum behavior arising at the event horizon. To see the universality of Hawking radiation, the DRS method have also been used to compute the Hawking temperature in terms of the two dimensional effective metric.

The covariant anomaly cancellation method has also been generalized to study the Hawking radiation of the spherically symmetric, asymptotically AdS black holes [4]. These black holes can be seen as the analogs of the usual Schwarzschild AdS black holes. We have obtained their Hawking temperature which recovers that of the asymptotically flat black holes in [2] when $\Lambda_{W}=0$.

Finally, we give a simple comment on the methods of anomaly cancellation and effective action. Both the methods are applicable to the theories that are generally covariant. Although the HL gravity theory is invariant under foliation preserving diffeomorphism, we can still use the two methods to study Hawking radiation of black holes in this gravity theory, since the group with foliation preserving diffeomorphism is a subgroup of the one with full diffeomporphism. Further investigation is required to prove the strict validity of applying 
both the methods to the gravity theory with foliation preserving invariance.

\section{Acknowledgments}

This work was partially supported by the Natural Science Foundation of China under Grant Nos. 10975058 and 10675051. J.J. Peng was also supported in part by a Graduate Innovation Foundation of Central China Normal University.

\section{References}

[1] P. Hořava, Phys. Rev. D 79, 084008 (2009), arXiv:0901.3775 [hep-th]; P. Horava, J. High Energy Phys. 03, 020 (2009), arXiv:0812.4287 [hep-th].

[2] A. Kehagias, K. Sfetsos, Phys. Lett. B 678, 123 (2009), arXiv:0905.0477 [hep-th].

[3] H. Lu, J.W. Mei, C.N. Pope, Phys. Rev. Lett. 103, 091301 (2009), arXiv:0904.1595 [hep-th].

[4] M.I. Park, J. High Energy Phys. 09, 123 (2009), arXiv:0905.4480 [hep-th].

[5] A. Ghodsi, E. Hatefi, arXiv:0906.1237 [hep-th]; M. Botta-Cantcheff, N. Grandi, M. Sturla, arXiv:0906.0582 [hep-th]; A. Ghodsi, arXiv:0905.0836 [hep-th]; E.Ó. Colgáin, H. Yavartanoo, J. High Energy Phys. 08, 021 (2009), arXiv:0904.4357 [hep-th]; R.G. Cai, L.M. Cao, N. Ohta, Phys. Rev. D 80, 024003 (2009), arXiv:0904.3670 [hep-th]; H. Nastase, arXiv:0904.3604 [gr-qc].

[6] Y.W. Kim, H.W. Lee, Y.S. Myung, Phys. Lett. B 682, 246 (2009), arXiv:0905.3423 [hep-th]; J.H. Chen, Y.J. Wang, arXiv:0905.2786 [gr-qc]; S.B. Chen, J.L. Jing, Phys. Rev. D 80, 024036 (2009), arXiv:0905.2055 [gr-qc]; S.B. Chen, J.L. Jing, arXiv:0905.1409 [gr-qc]; Y.S. Myung, arXiv:0906.0848 [hep-th]; Y.S. Myung, Phys. Lett. B 678, 127 (2009), arXiv:0905.0957 [hep-th].

[7] A. Castillo, A. Larranaga, arXiv:0906.4380 [gr-qc].

[8] R.G. Cai, L.M. Cao, N. Ohta, Phys. Lett. B 679, 504 (2009), arXiv:0905.0751 [hep-th]; Y.S. Myung, Y.W. Kim, arXiv:0905.0179 [hep-th]. 
[9] F.W. Shu, Y.S. Wu, arXiv:0906.1645 [hep-th]; G. Calcagni, arXiv:0905.3740 [hepth]; C. Charmousis, G. Niz, A. Padilla, P.M. Saffin, J. High Energy Phys. 08, 070 (2009), arXiv:0905.2579 [hep-th]; M. Li, Y. Pang, J. High Energy Phys. 08, 015 (2009), arXiv:0905.2751 [hep-th]; D. Orlando, S. Reffert, Class. Quantum Grav. 26, 155021 (2009), arXiv:0905.0301 [hep-th]; B.Chen, Q.G. Huang, Phys. Lett. B (in press), arXiv:0904.4565 [hep-th]; T.P. Sotiriou, M. Visser, S. Weinfurtner, JHEP 10, 033 (2009), arXiv:0905.2798 [hep-th]; S. Mukohyama, JCAP 09, 005 (2009), arXiv:0906.5069 [hep-th]; J. Kluson, JHEP 07, 079 (2009), arXiv:0904.1343 [hep-th].

[10] T. Takahashi, J. Soda, Phys. Rev. Lett. 102, 231301 (2009), arXiv:0904.0554 [hep-th].

[11] A.Z. Wang, Y.M. Wu, JCAP 07, 012 (2009), arXiv:0905.4117 [hep-th]; X. Gao, Y. Wang, R. Brandenberger, A. Riotto, arXiv:0905.3821 [hep-th]; C.J. Gao, arXiv:0905.0310 [astro-ph.CO]; Y.S. Piao, arXiv:0904.4117 [hep-th]; G. Calcagni, JHEP 09, 112 (2009), arXiv:0904.0829 [hep-th].

[12] S. Hawking, Nature (London) 248, 30 (1974); Commun. Math. Phys. 43, 199 (1975).

[13] S.P. Robinson, F. Wilczek, Phys. Rev. Lett. 95, 011303 (2005), gr-qc/0502074.

[14] S. Iso, H. Umetsu, F. Wilczek, Phys. Rev. Lett. 96, 151302 (2006), hep-th/0602146.

[15] S. Iso, H. Umetsu, Frank Wilczek, Phys. Rev. D 74, 044017 (2006), hep-th/0606018.

[16] K. Murata, J. Soda, Phys. Rev. D 74, 044018 (2006), hep-th/0606069.

[17] S. Iso, T. Morita, H. Umetsu, JHEP 04, 068 (2007), hep-th/0612286; Z.B. Xu, B. Chen, Phys. Rev. D 75, 024041 (2007), hep-th/0612261; H. Shin, W. Kim, J. High Energy Phys. 06, 012 (2007), arXiv:0705.0265 [hep-th]; W. Kim, H. Shin, J. High Energy Phys. 07, 070 (2007), arXiv:0706.3563 [hep-th]; A.P. Porfyriadis, Phys. Rev. D 79, 084039 (2009), arXiv:0811.2822 [hep-th]; A.P. Porfyriadis, Phys. Lett. B 675, 235 (2009), arXiv:0904.2042 [hep-th].

[18] L. Bonora, M. Cvitan, J. High Energy Phys. 05, 071 (2008), arXiv:0804.0198 [hepth]; L. Bonora, M. Cvitan, S. Pallua, I. Smolić, J. High Energy Phys. 12, 021 (2008), arXiv:0808.2360 [hep-th].

[19] S.Q. Wu, J.J. Peng, Class. Quantum Grav. 24, 5123 (2007), arXiv:0706.0983 [hep-th] 
[20] J.J. Peng, S.Q. Wu, Chin. Phys. B 17, 825 (2008), arXiv:0705.1225 [hep-th]; E. Papantonopoulos, P. Skamagoulis, Phys. Rev. D 79, 084022 (2009), arXiv:0812.1759 [hep-th]; S.W. Wei, R. Li, Y.X. Liu, J.R. Ren, Eur. Phys. J. C 65, 281 (2010), arXiv:0901.2614 [hep-th].

[21] S.Q. Wu, J.J. Peng, Z.Y. Zhao, Class. Quantum Grav. 25, 135001 (2008), arXiv:0803.1338 [hep-th].

[22] U. Miyamoto, K. Murata, Phys. Rev. D 77, 024020 (2008), arXiv:0705.3150 [hep-th]; B. Chen, W. He, Class. Quantum Grav. 25, 135011 (2008), arXiv:0705.2984 [gr-qc].

[23] R. Banerjee, S. Kulkarni, Phys. Rev. D 77, 024018 (2008), arXiv:0707.2449 [hep-th].

[24] J.J. Peng, S.Q. Wu, Phys. Lett. B 661, 300 (2008), arXiv:0801.0185 [hep-th].

[25] S. Gangopadhyay, S. Kulkarni, Phys. Rev. D 77, 024038 (2008), arXiv:0710.0974 [hepth]; S. Nam, J.D. Park, Class. Quantum Grav. 26, 145015 (2009), arXiv:0902.0982 [hep-th]; J.J. Peng, S.Q. Wu, Gen. Rel. Grav. 40, 2619 (2008), arXiv:0709.0167 [hepth].

[26] R. Banerjee, Int. J. Mod. Phys. D 17, 2539 (2009), arXiv:0807.4637 [hep-th]; R. Banerjee, B.R. Majhi, Phys. Rev. D 79, 064024 (2009), arXiv:0812.0497 [hep-th].

[27] R. Banerjee, S. Kulkarni, Phys. Lett. B 659, 827 (2008), arXiv:0709.3916 [hep-th]; R. Banerjee, S. Kulkarni, Phys. Rev. D 79, 084035 (2009), arXiv:0810.5683 [hep-th].

[28] H. Leutwyler, Phys. Lett. B 153, 65 (1985); 155, 469(E) (1985).

[29] A. Shirasaka, T. Hirata, arXiv:0804.1910 [hep-th].

[30] S. Gangopadhyay, Phys. Rev. D 77, 064027 (2008), arXiv:0712.3095 [hep-th]; S. Kulkarni, Class. Quantum Grav. 25, 225023 (2008), arXiv:0802.2456 [hep-th].

[31] T. Damour, R. Ruffini, Phys. Rev. D 14, 332 (1976); S. Sannan, Gen. Rel. Grav. 20, 239 (1988). 